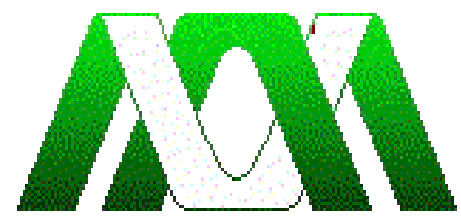

Casa abierta al tiempo

UNIVERSIDAD AUTÓNOMA METROPOLITANA

UNIDAD IZTAPALAPA

\title{
DIVISIÓN DE CIENCIAS SOCIALES Y HUMANIDADES
}

POSGRADO EN CIENCIAS ANTROPOLÓGICAS

Culturas y Músicas: Un acercamiento a la manera en que se construyen los gustos musicales.

Natalia Montes Marín

ENSAYO

Para obtener el Diploma de Especialización

en Antropología de la Cultura

Director: Dr. Néstor García Canclini

México, D.F.

Junio de 2017 


\section{CULTURAS Y MÚSICAS: UN ACERCAMIENTO A LA MANERA EN QUE SE CONSTRUYEN LOS GUSTOS MUSICALES. ${ }^{1}$}

\section{Resumen.}

Conceptos como Cultura, gustos, multiculturalismo, interculturalidad, campos, habitus entre otros, se indagan para revisar de qué manera se construyen los gustos musicales.

El uso de diversas teorías como: la Interculturalidad de Néstor García Canclini, La distinción o la teoría explicativa de los gustos de Pierre Bourdieu, la teoría del Actor Red de Latour y la teoría del Omnivorismo Cultural de Peterson, son herramientas teóricas para analizar cómo operan los modos de adquisición del capital cultural en relación a los niveles de educación y a los procesos de producción, circulación y consumo.

De esta manera, el capital cultural interacciona con: actores humanos y no humanos, redes socio-culturales, concepciones de la estética asociadas a las clases sociales, entre otros aspectos que serán útiles para ver la manera en que se edifican tendencias hacia determinados gustos.

En este sentido, la construcción de los gustos musicales se presentan como un reflejo de las diferencias, desigualdades y desconexiones, que giran entorno al capital escolar, simbólico y cultural.

\section{Palabras clave.}

Cultura, música, gustos musicales, educación, interculturalidad y clases sociales.

\footnotetext{
${ }^{1}$ Este ensayo fue presentado el 29 de junio del año 2017.
} 


\section{Introducción.}

La pregunta por el carácter distintivo de los gustos en el ámbito musical, es una cuestión bastante compleja. Autores como Denys Cuche, Thomas Hylland Eriksen, Claude Lévi-Strauss, Néstor García Canclini, Pierre Bourdieu, Bruno Latour, Carlos Fernández, Riie Heikkilä, Luis Reygadas, Robert Faulkner, Howard Becker, entre otros, han trabajo teorías y conceptos útiles para indagar en la identificación con determinadas preferencias musicales.

El propósito de este texto es acercarnos a la manera en que se construyen los gustos musicales. En este sentido, se identifican diversos factores que inciden en la inclinación hacia ciertas preferencias musicales y se abordan de manera teórica y práctica. La importancia de este escrito radica en que los gustos musicales es un tema que tiende a verse como innato del ser humano, pero su carácter sociocultural poco se explorado desde la disciplina antropológica.

Aunque este trabajo presenta algunas críticas a la teoría de los gustos de Bourdieu, no pretende desmeritar su propuesta. Por el contrario, la reconoce como pionera en el análisis de las relaciones entre los gustos, las clases sociales y el estatus social. Es una base para ampliar y seguir indagando acerca de los gustos musicales desde una perspectiva antropológica.

El texto está dividido en dos apartados: el primero contiene una revisión histórica del concepto de "Cultura" en torno a su relación con lo artístico y la educación. En el segundo señala la misma relación en referencia al concepto de "gustos". El grueso de este apartado es el análisis de la correlación que existe entre los gustos con: la manera en que se adquiere el conocimiento, la influencia de los niveles de educación, los problemas de la conceptualización en torno a lo que se considera "legítimo" o no y lo que se considera estético en el sentido de "bello" o "funcional", el papel que cumple la economía en la teoría y la práctica de acuerdo a los gustos, la influencia de los campos y habitus como determinantes en las preferencias musicales, la visión dinámica entre las prácticas y el consumo cultural de las clases sociales y finalmente como se manifiesta esto en la vida social. 
Por último, el ensayo presenta unas conclusiones que acogen los temas mas centrales para responder a la pregunta inicial: ¿Cómo se construyen los gustos musicales?. 


\section{Relación entre "Cultura", arte y educación.}

Hablar de "Cultura" ha denotado históricamente diferentes definiciones, como el cultivo de la tierra, el cultivo de una facultad o habilidad, el cultivo de ciertos conocimientos, entre otras definiciones que la relacionaron con el arte y la educación desde siglos atrás. Denys Cuche en su libro Las nociones de cultura en las ciencias sociales, realiza un recuento histórico de las concepciones ligadas al término, donde se observa su relación con el ámbito artístico en Francia y Alemania. Lo anterior se expone en el siguiente recuadro:

\begin{tabular}{|l|l|}
\hline \multicolumn{2}{|c|}{ Definiciones de la noción de "Cultura" en Francia y Alemania } \\
recopiladas por Denys Cuche.
\end{tabular}

\footnotetext{
${ }^{2}$ Denys Cuche, La noción de cultura en las ciencias sociales, Buenos Aires, Nueva Visión, Colección Claves, 1996, pp. 5-17.

${ }^{3}$ Ídem., p. 10.
} 


\begin{tabular}{|c|c|}
\hline & $\begin{array}{l}\text { pensarse como un estado de la mente cultivada por la } \\
\text { instrucción. Se relacionó con las ideas de progreso, razón, } \\
\text { evolución y educación. Además, se usaba en singular y era lo } \\
\text { opuesto a la noción de "Naturaleza". El concepto de Cultura } \\
\text { se adjudicó a un progreso individual y el de Civilización se } \\
\text { utilizó para referir un progreso colectivo. } \\
\text { En este siglo se consolida su sentido moderno. } \\
\text { Mediados y finales del siglo en Alemania: Los intelectuales } \\
\text { de la época diferenciaron entre los "valores corteses" de la } \\
\text { aristocracia, los cuales eran considerados como superficiales y } \\
\text { los "valores espirituales" como la ciencia, el arte, la filosofía y } \\
\text { la religión, considerados como auténticos. También criticaron } \\
\text { el desinterés de los gobernantes alemanes por el arte y la } \\
\text { literatura. } \\
\text { El concepto se expresa como una característica de la } \\
\text { burguesía intelectual alemana. No obstante, ser civilizado no } \\
\text { significaba que se tuviera cultura. En otras palabras, ser } \\
\text { refinado en las costumbres no implicaba estar instruido } \\
\text { educativamente. }\end{array}$ \\
\hline Siglo XIX & $\begin{array}{l}\text { En Francia: Se asocia con lo colectivo, inclusive se hablaba } \\
\text { de una "Cultura de la Humanidad". Había una visión } \\
\text { universalista del término que entendía el género humano } \\
\text { como una unidad. } \\
\text { En Alemania: Se convierte en una característica de la nación } \\
\text { porque expresa el alma del pueblo. Se opone a la Civilización } \\
\text { que refiere lo material, lo económico y lo técnico. "Cultura" se } \\
\text { define como un "Conjunto de conquistas artísticas, } \\
\text { intelectuales y morales que constituyen el patrimonio de una } \\
\text { nación [...]". }{ }^{4} \text { Se observa una visión particularista del } \\
\text { concepto, que reconoce la diversidad cultural y que será } \\
\text { fundamental para la creación de la constitución del Estado- } \\
\text { nación alemán. }\end{array}$ \\
\hline Siglo XX & $\begin{array}{l}\text { En Francia: Su asociación con lo colectivo se desequilibra. } \\
\text { Desde el siglo XIX hasta comienzos del siglo XX el término } \\
\text { alemán Kultur se tradujo en Francia como "Civilización". } \\
\text { En este siglo continúa el debate entre Francia y Alemania con } \\
\text { respecto a la visión universalista y particularista. Ambas } \\
\text { maneras de entender "Cultura", estarán vigentes hasta la }\end{array}$ \\
\hline
\end{tabular}

${ }^{4}$ Ídem., p.16. 
\begin{tabular}{|l|l|}
\hline & época contemporánea. \\
\hline
\end{tabular}

Cuadro 1. Elaboración propia a partir del texto: La noción de cultura en las ciencias sociales de Denys Cuche.

Este bosquejo histórico permite observar algunos cambios significativos entorno al concepto y su consonancia con el arte, la educación y las costumbres. Por eso el siglo de las luces resulta crucial para comprender esta asociación. Especialmente porque sigue vigente.

La noción científica de "Cultura" en las ciencias sociales ha sido bastante debatida y reformulada, pero claramente no ha logrado distanciarse de los problemas referentes a las dicotomías: Cultura/Civilización y Universalismo/Particularismo. Un ejemplo se observa en el trabajo de Tommas Hylland Eriksen, quien analizó y criticó el uso del concepto de "Cultura" en el informe de la UNESCO titulado Our Creative Diversity del año 1995, en base a los debates académicos conceptuales que había en la primera década del siglo XXI. El autor también se percata de algunas ambigüedades, omisiones y contradicciones que hay en el informe con respecto al término. "Cultura" se presentó primero en relación al trabajo artístico o como un modo de vida, y segundo, como producción artística; las culturas fueron vistas como arraigadas, viejas y aisladas, omitiendo los fenómenos culturales modernos. Además, "Cultura" y "Etnicidad" se expresan sin diferenciación, y no se indaga mayormente en el concepto de "Identidad". ${ }^{5}$ Otro ejemplo que expresa la continuidad de estos debates se puede observar en el texto que la UNESCO le encargó a Lévi-Strauss a mediados del siglo XX, titulado: Raza e Historia. A grandes rasgos, el escrito trata de un discurso antirracista basado en el análisis de aspectos concernientes a la diversidad cultural, el etnocentrismo, el evolucionismo biológico y social, y las ideas de progreso y civilización. ${ }^{6}$ Así pues, la noción de

\footnotetext{
${ }^{5}$ Vid. Thomas Hylland Eriksen, "Between universalism and relativism: a critique of the UNESCO concept of culture", en: Jane K. Cowan, Marie-Bénédicte Dembour and Richard A. Wilson (editores), Culture and Rights. Anthropological Perspectives, Cambridge, UK, 2008, pp. 127-148, recuperado el 7 de junio de 2017, en: http://hyllanderiksen.net/Rights.pdf

${ }^{6}$ Vid. Claude Levi-Strauss Raza e Historia, Altaya, Madrid, [(1971) 1999], pp. 37-104, recuperado

el 9 de junio de 2017, en:
} 
"Cultura" como recurso para el desarrollo, la gestión del patrimonio y la diversidad, fue cuestionada bajo los discursos científicos sociales del momento.

Néstor García Canclini considera que para lograr una definición sociosemiótica de la "Cultura" en las ciencias sociales, hay que lograr un consenso entre las narrativas del uso coloquial que distinguen "Cultura" de "Civilización" y entre las narrativas del uso científico que oponen los conceptos de "Naturaleza" y "Sociedad" contra el concepto de "Cultura". En este sentido, el autor propone la siguiente definición: "La cultura abarca el conjunto de los procesos sociales de significación, o de un modo más complejo, la cultura abarca el conjunto de procesos sociales de producción, circulación y consumo de la significación en la vida social."7 La crítica que recibe esta definición es que no abarca lo que constituye a cada cultura. Esto se puede reflexionar desde las diferentes narrativas que hay entorno a la función social y el sentido que tiene la cultura dentro de la sociedad. Es decir, la "Cultura" puede ser entendida como organizadora de identidad $^{8}$; como una instancia simbólica de producción y reproducción de la sociedad; como un espacio conformado por consensos y hegemonías que configuran la política y la legitimidad ${ }^{9}$; y/o como una dramatización eufemizada de los conflictos sociales que representa simbólicamente lo que está en medio de las diversas prácticas culturales. ${ }^{10}$ Es este sentido, es indispensable abordar las interacciones interculturales que

http://eva.universidad.edu.uy/pluginfile.php/307211/mod_resource/content/1/28\%20\%28Unidad\%2 05\%29.pdf

${ }^{7}$ Néstor García Canclini, Diferentes, desiguales y desconectados. México, Gedisa, 2005, p. 34.

${ }^{8}$ Stuart Hall expone la dificultad que implica hablar de "Identificación" e "identidad". No obstante, a partir de sus argumentos se puede definir "la identificación" como un proceso constante y ambiguo que interacciona con las diferencias y las fronteras simbólicas. En ese proceso se puede permanecer o no. En la misma dirección, "las identidades" se construyen desde el poder y la exclusión, son temporales, inestables, tienen su origen en la diferencia y se conciben como una unidad que demarca fronteras entre lo interno y lo externo de ellas. Vid. Stuart Hall, "Introducción: ¿quién necesita "identidad"?", en: Cuestiones de identidad cultural, Stuart Hall y Paul du Gay (comp.), Buenos Aires, Amorrortu, 2003, pp. 13-39.

${ }^{9}$ La legitimidad se presenta como un proceso evaluativo a cargo de actores específicos que definen si determinadas acciones deben ser sancionadas o justificadas. En este sentido, la justificación proporciona legitimidad. Vid. Arthur Tuden, Marc J. Swartz, Víctor W. Turner, Reseña de: "'Introducción" a Political Anthropology" de Marc J. Swartz, Víctor W. Turner y Arthur Tuden, en: Alteridades, vol. 4, núm. 8, 1994, pp.106-107, recuperado el 12 de junio de 2017, en: http://www.redalyc.org/pdf/747/74711353010.pdf

${ }^{10}$ Néstor García Canclini, op. cit., pp. 29-43. 
transforman las nociones de "Cultura" e "Identidad", y las interacciones simbólicas que dotan de sentido a lo material.

Coincido con Canclini en que hablar de "Cultura" involucra dos modos de producción de lo social: 1) la multiculturalidad que resalta y acepta la heterogeneidad o la diversidad cultural, y 2) la interculturalidad entendida como las relaciones e intercambios entre grupos que remiten a la confrontación, el conflicto y/o la negociación. En base a la teoría de la interculturalidad propuesta por el autor, se puede argumentar que los gustos musicales se construyen a partir de los procesos relacionados con: 1) las diferencias en las prácticas culturales que se expresan en las lenguas, las clases sociales, entre otras; 2) las desigualdades socio-económicas que implican inserción o exclusión, y 3) las desconexiones provocadas por la dificultad de ser incluidos debido a discriminaciones, desempleo, desplazamiento, etc. ${ }^{11}$

En base a todo lo anterior, la noción de "Cultura" ligada al progreso, al intelectualismo, al arte y a la educación sigue latente, y debe pensarse en relación con los problemas de exclusión, diferencia y desigualdad en el acceso del capital cultural, escolar y simbólico. Todo esto será elemental para responder a la pregunta por el cuándo y cómo se construyen los gustos musicales.

${ }^{11}$ Ídem., pp. 45-82. 


\section{"Dime qué gustos musicales tienes y te diré a qué clase social perteneces"12}

La importancia del apartado anterior, radica en que las nociones de "Cultura" y "gustos" tienen en común una fuerte afinidad con la multiculturalidad, la interculturalidad, lo artístico y educativo. Pierre Bourdieu en su libro La distinción ${ }^{13}$, estudió la correlación que hay entre los gustos y las clases sociales a partir del análisis de las relaciones entre las prácticas culturales y el capital escolar -que corresponden al nivel educativo o al conocimiento adquirido tardíamente- y las relaciones entre las prácticas culturales y el capital de origen social -que corresponde a los saberes heredados por la familia-.

Bourdieu y Canclini ${ }^{14}$ coinciden en que los gustos expresan diferencias sociales que obedecen a la forma de adquisición y uso del capital cultural ${ }^{15}$, y a la manera en que éste opera dentro de los diferentes mercados (escolares o extraescolares). Esto se puede pensar desde el ámbito musical y desde otras esferas más.

Pierre Bourdieu distingue tres universos de gustos asociados a las prácticas culturales y al origen social o escolar del capital cultural: "legítimo" o burgués; medio y popular. Denomina el primer "universo de gustos" como "legítimo", justificado en el gusto por las "obras legítimas". Él menciona que "toda obra legítima tiende en realidad a imponer las normas de su propia percepción, y que define tácitamente como único legítimo el modo de percepción que establece cierta disposición y cierta competencia". ${ }^{16}$ Además, afirma que las obras legítimas no consideran si los diferentes agentes cuentan con los mismos medios para

\footnotetext{
${ }^{12}$ En base al refrán "Dime con quién andas y te diré quién eres", construí este porque congenia con el análisis premonitorio acerca del gusto propuesto por Bourdieu en relación a las clases sociales, el cual se pondrá en discusión.

${ }^{13}$ Pierre Bourdieu, La distinción. Criterio y bases sociales del gusto, Madrid, Taurus, 1991, pp. 932; 61-94.

${ }^{14}$ Néstor García Canclini, "La sociología de la cultura de Pierre Bourdieu" en: Pierre Bourdieu, Sociología y cultura, México, Grijalbo, 1990, p. 29.

${ }^{15}$ El capital cultural es el producto garantizado por medio de la transmisión familiar o a través de la escuela.

${ }^{16}$ Pierre Bourdieu, op. cit., p. 26.
} 
cumplirla. Sin embargo, aquí hay un problema. En el momento que denomina a este primer universo de gustos como "legítimo", reproduce una idea de superioridad que posiciona una vez más aquellos gustos de las clases altas, contraponiendo y sacrificando implícitamente la legitimidad de los gustos de las demás clases sociales.

En este sentido, considero que Bourdieu es presa de la tercera ausencia en la investigación de las inequidades expuestas por Luis Reygadas. Es decir, la ausencia o el papel secundario de los procesos simbólicos que cuestionan la desigualdad. Aquí la tendencia es la siguiente:

La cultura suele aparecer como algo homogéneo, como una fuerza unificadora, que cumple la función de estabilizar y hacer corresponder los valores y deseos de los individuos con los requerimientos estructurales del sistema. Ya sea que esta coincidencia entre valores y estructura social se vea como algo positivo, (porque permite el consenso y la estabilidad) o como algo negativo (porque expresa el predominio ideológico de la clase dominante y contribuye a reproducir relaciones sociales injustas), el caso es que suele darse por sentada la correspondencia entre la estructura social inequitativa y la manera de pensar y los valores de las personas. Unos la celebran y otros la deploran, pero tiende a sobreestimarse la capacidad que tiene la cultura para legitimar, naturalizar, estabilizar y reproducir las desigualdades. Suele concederse poca importancia a otras prácticas simbólicas que critican la desigualdad, que cuestionan que sea algo natural, que promueven mayor igualdad. ${ }^{17}$

En este orden de ideas, el autor subestimó la capacidad de los demás "universos de gustos" para legitimar. Si la legitimidad es dinámica y se construye en los "campos" o espacios sociales, no veo una razón objetiva para clasificar un gusto como "legítimo" asociado a una clase social en específico. Entonces si se me permite, Bourdieu reproduce un "gustocentrismo clasista". De esta manera concuerdo con Canclini en hablar de un "universo burgués". La crítica también es

\footnotetext{
${ }^{17}$ Luis Reygadas, "Más allá de la legitimación: Cinco procesos simbólicos en la construcción de la igualdad y la desigualdad", en: Mayarí Castillo y Claudia Maldonado (eds.) Desigualdades: Tolerancia, legitimación y conflicto en las sociedades latinoamericanas, RIL. Editores, Santiago de Chile, p. 44.
} 
aplicable cuando se habla de "obras legítimas" asociadas únicamente a la clase burguesa. Lo que es indiscutible, es que los gustos que caracterizan este primer universo (conocimiento de cine, jazz, canción, entre otros.) aumentan de acuerdo al nivel escolar.

El segundo "universo de gustos" denominado "medio" no es muy indagado, pero el autor lo distingue por la tendencia que tiene a crear y/o escuchar obras menores de las artes mayores. Hay que prestar mucha atención a este tipo de comparaciones porque en ellas se expresa nuevamente lo que se considera legítimo y lo que no. La postura que se tiene cuando se compara, también está inmersa en la clasificación. La clase media podría problematizarse mucho más, ya que se presenta como una "frontera difusa" donde fluctúan los gustos musicales de los otros dos "universos de gustos". Bourdieu solo la analiza en relación a la clase burguesa y la presenta como si no tuviera "originalidad". El hecho de que se apropien de obras musicales como la Rapsodia húngara se atribuye al deseo de querer imitar los gustos de la clase burguesa. No obstante, la creación y/o apropiación de otros gustos musicales queda en cuestión y estará bastante justificado desde el "omnivorismo cultural" como veremos más adelante. Visto de esta manera, también valdría la pena revisar las clasificaciones de los gustos que se legitiman dentro de la clase media en relación con el universo popular. Es decir, hace falta pensar la construcción de los gustos musicales en otras direcciones, desde todos sus "matices".

El tercer "universo de gustos" denominado "popular", se distingue por las preferencias en la llamada música "ligera" o comercial y en la llamada música "culta" desvalorizada por su amplia divulgación. En este universo vale la pena resaltar la propuesta de Panofsky acerca de la concepción estética que considera una obra de arte desde una percepción únicamente "práctica", en oposición a la visión objetiva que determina que la disposición estética tiene el poder para considerar lo que es o no es arte. ${ }^{18}$

\footnotetext{
${ }^{18}$ Pierre Bourdieu, op. cit., pp. 26-27.
} 
García Canclini ${ }^{19}$ expone algunos tipos de estéticas que complementan todo lo anterior. 1) la estética dominante como aquella que exige una disposición más educada y que a su vez integra la estética incestuosa -ejemplificada como el arte por y para los artistas-; 2) la estética de los sectores medios conformada por la industria cultural y prácticas como la fotografía; y 3) la estética popular que privilegia lo funcional. En este sentido, el autor expresa: "La distinción acumula ejemplos semejantes para argumentar que el consumo popular se opone al burgués por su incapacidad de separar lo estético de lo práctico."20

Esta oposición va a relacionar "lo estético" o "bello" con la burguesía y "lo práctico o funcional" con las clases populares. El sentido práctico no desplaza siempre y/o necesariamente las preferencias por determinados gustos musicales. Más bien está yuxtapuesto o inmerso en los gustos. Por ejemplo, el músico y compositor chocoano Octavio Panesso expresa esta dicotomía entre los gustos musicales y el sentido práctico:

[...] a mi me gustaría hacer música tradicional, pero cuando yo mismo estoy poniendo la plata, cuando de mi sueldo estoy utilizando los recursos, me veo prácticamente obligado a hacer chirimía moderna ${ }^{21}$, a hacer una música con tendencias modernas y para el mundo, sin perder lógicamente la tradición que eso es lo que le regalo a mi pueblo, que eso es lo que le regalo al proyecto, fusionarla y que siempre la chirimía esté presente.

Pero hoy por hoy quién va a meter tanta plata de su bolsillo para sostener un proyecto que debe ser del gobierno, que debe ser del gobernador, que debe ser de la alcaldía. Hay que fusionarla por ejemplo a nivel instrumental, ya es meterle un piano, meterle un bajo, meterle una guitarra, meterle cualquier otro ritmo. Lógicamente yo la sigo llamando chirimía cuando veo el clarinete y veo los aires [...] la chirimía está ahí, está en el ambla de lo que nosotros hacemos, pero lo hacemos

\footnotetext{
${ }^{19}$ Néstor García Canclini, op. cit., 2005, pp. 13-82.

${ }^{20}$ Ídem., pp. 61-69.

${ }^{21}$ En Quibdó, municipio y capital del departamento del Chocó en Colombia, hay un tipo de agrupación de música tradicional llamado "chirimía". Su formato instrumental consta de clarinete, bombardino, saxofón (opcional), redoblante, platillos y bombo. Lo que Panesso denomina como "chirimía moderna" hace referencia a formatos emergentes que han surgido en relación a la chirimía tradicional, que integran instrumentos que no corresponden al formato tradicional, pero que buscan legitimidad por medio de definiciones como: tecnochirimías, chirimías ampliadas, papayeras, entre otras.
} 
por cuestiones de rentabilidad y para que nuestros proyectos sigan vivos. De pronto el día que seamos subsidiados por el Estado, por una nación, por una comunidad internacional, haremos la música que ellos quieren y como la quieren. [...] por eso es que la música se ha proliferado tanto, porque los pelaos aún no tienen apoyo y quieren el apoyo del papá y la mamá les consigan los primeros instrumentos y ellos a partir de ahí van a hacer lo que les da la gana, porque cuando no hay ni control [...] entonces ellos qué hacen, aprovechan y producen lo que la gente ha querido llamarle papayera o cualquier otra cosa. ${ }^{22}$

Si bien la tensiones económicas contribuyen a que haya una negociación con los gustos musicales y la creación musical, también corresponden a un problema enraizado en la estructura social. Tanto el sentido estético como el práctico puede verse reflejado en este universo de gustos populares. Por ejemplo, puede predominar la importancia de que se oiga "bonito" aunque no se tenga conocimiento teórico con respecto a la composición. También puede predominar el hecho de componer melodías o letras "contagiosas" y repetitivas que se divulguen y se vendan fácil y rápido, aunque no sean muy afines con los gustos.

Indiscutiblemente muchas de las relaciones presentes en esos tres universos de gustos musicales, son de vieja data. No es fortuito que hasta la fecha se siga asociando y reproduciendo por una parte, un pensamiento que conecta la música clásica con: "lo erudito", "lo estético", "lo culto", "lo legítimo" y las clases "altas" y por otra parte, un pensamiento que vincula la música tradicional-popular con: "lo empírico", "lo práctico", "lo vulgar", "lo ilegítimo" y las clases "populares".

Así como la definición de "Cultura musical" que propone Pierre Bourdieu reúne conocimientos, experiencias y espiritualismo, ${ }^{23}$ las características otorgadas a esos universos de gustos también se ven impregnadas por estos aspectos. En este sentido los conceptos bourderianos de "campos" y "habitus" son una base para comprender de qué manera se construyen los gustos musicales.

\footnotetext{
22 Octavio Panesso Arango, entrevista realizada el 30 de Septiembre del año 2012, por: Natalia Montes Marín, Quibdó, Chocó, Colombia.

23 Pierre Bourdieu, op. cit., p. 16
} 
Los "campos" son entendidos como espacios sociales que pueden ser esferas profesionales, académicas, políticas, entre otras, en las que se desempeñan los participantes o agentes. Y los "habitus" hacen referencia a los modos de pensar y actuar en relación a los campos; son sistemas de disposiciones, transferibles entre los sujetos que condicionan el desarrollo de los gustos. ${ }^{24}$

Amén a lo anterior, la relación entre campos, habitus, adquisición de capital (cultural y simbólico), producción, circulación, consumo y diferencias sociales, están presentes en la construcción de los gustos musicales. Los niveles de instrucción adquirida o de origen social pueden explicar el origen de los habitus cultivados y serán vitales para comprender los usos del capital, los modos de producción, circulación y los valores en los mercados para su consumo.

Concuerdo con que la música occidental asociada a "lo culto", a los campos profesionales, a la instrucción expresada en la lectura musical y al conocimiento de los convencionalismos de la escritura musical, puede provocar unas preferencias por determinados tipos de música. Pero sucede lo mismo con la música popular, que desde el empirismo también genera tendencias por determinados gustos. No obstante, hay que ser cuidadosos para no caer en un determinismo de gustos estáticos o demasiados difusos entre las clases.

Las características entre las clases sociales no deben verse como estáticas. Tanto para Eric Wolf ${ }^{25}$ como para José Luis Molina ${ }^{26}$, el análisis de las redes personales y sus relaciones, ayudan a identificar y explicar fenómenos sociales en los niveles micro y macro. Es decir, entre las interacciones individuales y su relación con las instituciones y estructuras sociales. Esta propuesta se complementa con la Teoría del Actor Red propuesta por Bruno Latour que analiza -a grandes rasgos- las relaciones dinámicas entre actores humanos y actores no humanos en pro de

\footnotetext{
24 Pierre Bourdieu, op. cit., pp. 9-32; 61-94.

${ }^{25}$ Eric Wolf, "Relaciones de parentesco, de amistad y de patronazgo en las sociedades complejas" en: Eric Wolf, (et al.), Antropología Social de las sociedades complejas, Alianza Editorial, 1990, pp.19-39.

${ }^{26}$ José Luis Molina, "El estudio de las redes personales; contribuciones, métodos y perspectivas", en: Empiria, Revista Metodológica de las Ciencias Sociales, núm. 10, julio-diciembre, 2005,
} 
reensamblar lo social. Es decir, hay una necesidad de reunir instituciones, procedimientos y conceptos que sean capaces de volver a relacionar lo social. ${ }^{27}$

En la teoría explicativa de los gustos de Bourdieu, se pueden identificar conexiones de este tipo para el análisis musical. Por ejemplo, la relación que tiene un actor humano con las instituciones sociales (familia) o las instituciones académicas (universidades), en consonancia con: los procedimientos, modos de adquisición u origen (social o escolar) del capital cultural; el uso que se hace del capital en otras esferas institucionales (mercados globales); las interacciones con actores no humanos (instrumentos musicales, cd's, computadoras, etc.); la carga simbólica de estas interacciones en relación a las diferencias sociales; y las propuestas conceptuales como: "habitus", "campo" y "gustos musicales", entre otros más.

Estas propuestas relacionales rememoran una afirmación que hace Bourdieu:

\section{[...] no existe nada por lo que permita tanto a uno afirmar su "clase" como los} gustos musicales, nada por lo que se sea tan infaliblemente calificado, es sin duda porque no existe práctica más enclasante, dada la singularidad de las condiciones de adquisición de las correspondientes disposiciones, que la frecuentación de conciertos o la práctica de un instrumento de música "noble" (menos generalizadas, permaneciendo constante todo lo demás, que la frecuentación del teatro, de los museos o incluso de las galerías de arte). ${ }^{28}$

En base a ella, se expone la siguiente cuestión: ¿qué tan cierto puede ser, que las diferencias de clase sean predecibles de acuerdo a los gustos musicales?. No está en cuestión que los gustos musicales expresan diferencias sociales, sino que a partir de los gustos se pueda determinar la pertenencia a una clase social específica.

\footnotetext{
${ }^{27}$ Bruno Latour, Changer de société-Refaire de la sociologie. Éditions La Découverte, París, 2006. Reasambling The Social. An Introduction to Actor-Network Theory, Oxford University Press, 2005. En español: Reemsamblar lo social. Una introducción a la teoría del actor red. Argentina, Manantial, 2008, pp. 25-27.

${ }^{28}$ Pierre Bourdieu, op. cit., p. 20. La negrilla es mía.
} 
Desde la teoría del omnivorismo cultural, la respuesta sería negativa principalmente porque en ella no hay una terna en la división de clases, más bien hay muchas posibilidades. Las interacciones sociales son más dinámicas y no están separadas drásticamente como en la teoría explicativa de los gustos de Bourdieu. Carlos Fernández y Riie Heikkilä proponen un debate muy interesante en torno al omnivorismo cultural. Éste critica la teoría bourdieriana de la distinción porque presenta las preferencias culturales entre las clases como estáticas y estrictamente separadas.

En este sentido, el vínculo entre el "omnivorismo cultural" y la variedad de estilos de vida, los diversos tipos de consumidores culturales, las consecuencias de las desigualdades sociales y las distinciones basadas en la lógica de la dominación, también resultan de gran utilidad ${ }^{29}$

El "omnivorismo cultural" hace referencia al que devora de todo. Una de las definiciones más abarcadoras del término, fue realizada por un grupo de investigación británico que la explica como un sector de la población de los países occidentales que se interesa por una gran variedad de formas de cultura y manifiesta mayor tolerancia social a la diversidad cultural y a los gustos en general. ${ }^{30}$ Este omnivorismo se impuso ante otros tipos de consumidores.

Las categorías de unívoros, omnívoros, inactivos y paucívoros presentadas por $\mathrm{R}$. A. Peterson en el año 2005, son una fuente interesante para pensar ¿cómo interactúan los consumidores con el capital cultural?. Las características de cada categoría reflejan interacciones interculturales y simbólicas con respecto a ese capital, como se puede observar en la siguiente tabla:

\footnotetext{
${ }^{29}$ Carlos Jesús Fernández Rodríguez y Riie Heikkilä, "El debate sobre el omnivorismo cultural. Una aproximación a nuevas tendencias en sociología del consumo", en: Revista Internacional de Sociología, Vol. 69, núm. 3, Septiembre-Diciembre, Madrid, 2011, Pp. 585-587.

${ }^{30}$ Ídem., pp. 589.
} 


\begin{tabular}{|l|l|}
\hline \multicolumn{2}{|c|}{ Propiedades de los diferentes tipos de consumidores culturales } \\
propuestas por Peterson
\end{tabular}

Cuadro 2. Elaboración propia a partir del texto "El debate sobre el omnivorismo cultural. Una aproximación a nuevas tendencias en sociología del consumo" de Carlos Jesús Fernández Rodríguez y Riie Heikkilä. ${ }^{31}$

De cualquier manera, la teoría del omnivorismo cultural también defiende que las preferencias por determinados gustos musicales tienen una correlación con el origen del capital cultural en relación a los campos, los habitus, las interacciones interculturales y simbólicas en las prácticas culturales.

En el libro titulado: El jazz en acción: la dinámica de los músicos sobre el escenario escrito por Robert Faulkner y Howard Becker ${ }^{32}$, hay un interés por saber ¿cómo hacen música los músicos?. Para responder a esta pregunta trabajaron tres casos etnográficos que reflejan diversas dinámicas y relaciones que hemos venido abordando, como son: la diferencia de gustos entre las clases sociales, la

\footnotetext{
${ }^{31}$ Ídem., pp. 597-593.

${ }^{32}$ Robert Faulkner y Howard Becker, El jazz en acción: la dinámica de los músicos sobre el escenario, Argentina, Siglo XXI, 2011.
} 
identificación con determinados géneros musicales en relación al capital cultural y su correlación con el campo y el habitus, las relaciones entre actores humanos y no humanos, el uso y consumo del capital cultural, entre otros.

El primer caso etnográfico es de 1951. Becker describe que tuvo lugar en un bar llamado "Club 504" que estaba ubicado en un barrio popular de Chicago. Él tocaba el piano en un trío musical. Aunque tenían conocimiento de un amplio repertorio, los gustos musicales del patrón del bar, del público que lo frecuentaba y de los músicos que tocaban, no coincidian. Esto era suficiente para que el patrón y el público regularmente ignoraran la música del trío y para que los músicos se rehusaran a tocar los temas que en ocasiones les pedía público. Ellos nunca ensayaban, entonces se podían de acuerdo en el mismo momento en que se iba a tocar.

Entre Becker y Faulkner resumen un segundo caso etnográfico de Bruce MacLeod entre los años 70's y 80's acerca del -club date- ubicado en una región de Nueva York. Allí, ciertos líderes observaban el tipo de canciones que le gustaba bailar a la gente con la finalidad de que los músicos no tocaran canciones poco conocidas. Como el público normalmente solicitaba que se tocaran algunas obras específicas, los músicos tenían un amplio repertorio y varias estrategias para complacer al público. Constantemente tocaban arreglos musicales e improvisaban para cubrir las 3 o 4 horas seguidas que cubría una rutina.

Faulkner describe un tercer caso etnográfico del año 2007 basado en la experiencia dentro de un restaurante de Nueva Inglaterra. En este caso, había una un gusto compartido por el jazz entre los propietarios del recinto, el público y los músicos. En este lugar los músicos cuidaban su técnica a la hora de tocar.

Estos tres casos etnográficos permiten observar que la construcción de los gustos musicales resultan de todo un entramado socio-cultural que se expresa constantemente en las prácticas e interacciones culturales. Finalmente, cada uno 
de estos casos, refleja el encuentro entre agentes de diferentes clases sociales que se diferencian o coinciden en los gustos musicales de acuerdo a su capital cultural y las relaciones que conlleva hablar del mismo.

\section{Conclusiones.}

La noción de "Cultura" entendida como el estado o la acción de cultivar una habilidad no ha perdido el sentido. Por el contrario, sigue expresándose en términos del arte y la educación. La ambigüedad del término se ha prestado para mucha discusión, sin embargo su referencia con respecto a la diversidad cultural, la identidad, las interacciones interculturales y simbólicas, los procesos de producción, circulación y consumo, se han ido posicionando cada vez más en su definición. Estos aspectos nos remiten a los problemas de exclusión, diferencia y desigualdad en el acceso del capital escolar, simbólico y cultural.

Cuando exploramos el concepto de "gustos" y la manera en que opera a nivel social, se expone su afinidad con las definiciones y problemas que enfrenta también el concepto de "Cultura". La relación de los "campos" como espacios sociales y los habitus como modos de pensar y actuar en los "campos", se presentan como sistemas de disposiciones que condicionan la construcción y preferencia por ciertos gustos musicales. Amén a los anterior, las clases sociales deben verse de una manera menos estática y más interrelacionadas. No se pueden atribuir gustos determinados a clases sociales específicas, pues hay un flujo de interacción social que es dinámica. La teoría del omnivorismo cultural, la teoría de la interculturalidad y la teoría del actor red son indispensables para pensar en los flujos dinámicos de las relaciones sociales y las prácticas culturales que se reflejan en las inclinaciones por determinadas tipos de música.

Finalmente, se puede concluir que la construcción de los gustos musicales es un resultado de: 1) Diferencias en las prácticas culturales que surgen en relación a los espacios en que interactuamos y a los modos de vida. Estas prácticas influyen 
fuertemente en el origen de la adquisición del capital cultural. 2) Desigualdades socio-económicas que operan de una manera dinámica y se expresan en la interacción entre las clases sociales a nivel simbólico, económico y cultural. Estas desigualdades también se reproducen en base a las diferencias en las prácticas culturales.

3) Conexiones y desconexiones de los agentes sociales que se manifiestan en sintonía con las prácticas sociales y la adquisición del capital escolar, simbólico y cultural. Los casos etnográficos que se presentan son una prueba de ello. Reflejan que los modos de adquisición del capital cultural no condicionan el uso que se le da, pero si son una clave de conexión o desconexión entre agentes sociales diferentes y desiguales.

Las diversas relaciones que se hacen en este ensayo, expresan una gran diversidad de matices que desentrañan la manera en que se construyen los gustos musicales y que nos recuerdan que:

“No hay nada puramente natural en el hombre"33

${ }^{33}$ Cuche, Op. Cit., p. 6 


\section{Bibliografía.}

Bourdieu, Pierre, La distinción. Criterio y bases sociales del gusto, Madrid, Taurus, 1991, pp. 9-32; 61-94.

Cuche, Denys, La noción de cultura en las ciencias sociales, Buenos Aires, Nueva Visión, Colección Claves, 1996, pp. 5-62.

Faulkner, Robert y Becker, Howard, El jazz en acción: la dinámica de los músicos sobre el escenario, Argentina, Siglo XXI, 2011.

Fernández Rodríguez, Carlos Jesús y Heikkilä, Riie, "El debate sobre el omnivorismo cultural. Una aproximación a nuevas tendencias en sociología del consumo", en: Revista Internacional de Sociología, Vol. 69, No. 3, SeptiembreDiciembre, Madrid, 2011, pp. 585-606.

García Canclini, Néstor, Diferentes, desiguales y desconectados. México, Gedisa, 2005, pp. 13-82.

García Canclini, Néstor, "La sociología de la cultura de Pierre Bourdieu" en: Pierre Bourdieu, Sociología y cultura, México, Grijalbo, 1990, pp. 1-9; 13-32.

Hall, Stuart, "Introducción: ¿quién necesita "identidad"?, en: Cuestiones de identidad cultural, Stuart Hall y Paul du Gay (comp.), Buenos Aires, Amorrortu, 2003, pp. 13-39.

Hylland Eriksen, Thomas, "Between universalism and relativism: a critique of the UNESCO concept of culture", en : Jane K. Cowan, Marie-Bénédicte Dembour and Richard A. Wilson (editores), Culture and Rights. Anthropological Perspectives, Cambridge, UK, 2008, pp. 127-148, recuperado el 10 de junio de 2017, en: http://hyllanderiksen.net/Rights.pdf 
Latour, Bruno, Changer de société-Refaire de la sociologie. Éditions La Découverte, París, 2006. Reasambling The Social. An Introduction to ActorNetwork Theory, Oxford University Press, 2005. En español: Reemsamblar lo social. Una introducción a la teoría del actor red. Argentina, Manantial, 2008,

Levi-Strauss, Claude, Raza e Historia, Altaya, Madrid, [(1971) 1999], pp. 37-104, recuperado el 9 de junio de 2017, en: http://eva.universidad.edu.uy/pluginfile.php/307211/mod_resource/content $/ 1 / 28 \% 2$ 0\%28Unidad\%205\%29.pdf

Molina, José Luis, "El estudio de las redes personales; contribuciones, métodos y perspectivas", en: Empiria, Revista Metodológica de las Ciencias Sociales, núm. 10, julio-diciembre, 2005, pp. 71-105

Reygadas, Luis, "Más allá de la legitimación: Cinco procesos simbólicos en la construcción de la igualdad y la desigualdad", en: Mayarí Castillo y Claudia Maldonado (eds.) Desigualdades: Tolerancia, legitimación y conflicto en las sociedades latinoamericanas, RIL. Editores, Santiago de Chile, pp.39-68.

Tuden, Arthur; Swartz, Marc J. y Turner, Víctor W., "Reseña de: "Introducción" a Political Anthropology" de Marc J. Swartz, Víctor W. Turner y Arthur Tuden, en: Alteridades, vol. 4, núm. 8, 1994, pp. 106-107, recuperado el 12 de junio de 2017, en: http://www.redalyc.org/pdf/747/74711353010.pdf

Wolf, Eric, "Relaciones de parentesco, de amistad y de patronazgo en las sociedades complejas" en: Eric Wolf, (et al.), Antropología Social de las sociedades complejas, Alianza Editorial, 1990, pp.19-39.

\section{Entrevistas.}

Panesso Arango, Octavio, entrevista realizada el 30 de Septiembre del año 2012, por: Natalia Montes Marín, Quibdó, Chocó, Colombia. 
A4I

Casa abierta al tiempo

UNIVERSIDAD AUTONOMA METROPOLITANA-IZTAPALAPA

DIVISIÓN DE CIENCIAS SOCIALES Y HUMANIDADES

POSGRADO EN CIENCIAS ANTROPOLOGICAS

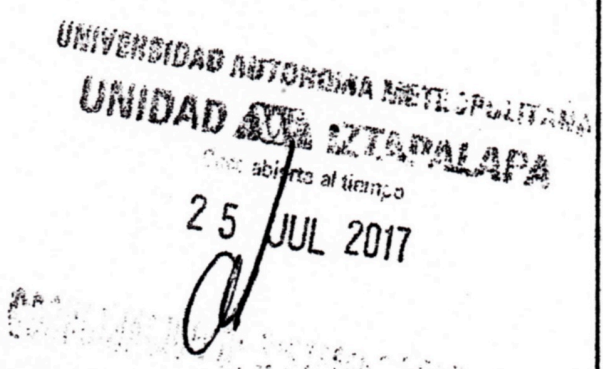

CONSTANCIA DE EVALUACIÓN DEL ENSAYO

PARA LA OBTENCIÓN DEL DIPLOMA EN LA ESPECIALIZACIÓN EN ANTROPOLOGÍA DE LA CULTURA

ALUMNA: MONTES MARÍN NATALIA

MATRICULA: 2163801332

TRIMESTRE 17-P

DIRECTOR: NÉSTOR RAÚL GARCÍA CANCLINI

LA ALUMNA PRESENTó EL ENSAYO TITULADO:

CULTURAS Y MÚSICAS: UN ACERCAMIENTO A LA MANERA

EN QUE SE CONSTRUYEN LOS GUSTOS MUSICALES.

OBTENIENDO LA CALIFICACIÓN DE:

\begin{tabular}{|l|l|l|}
\hline DIA & MES & ANO \\
19 & 07 & 2017 \\
\hline
\end{tabular}

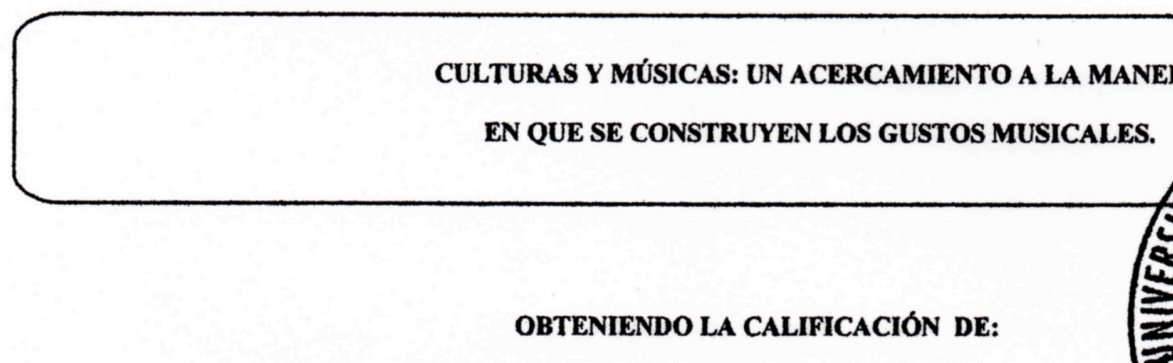

TONOMA
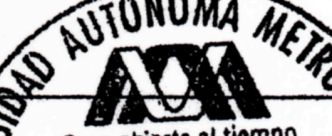

Casa abierta al tiempo

19 JUL 2017

C. S. H.

APROBAR (X)

NO APROBAR

(POSGRADO EN CIENCIAS

VID

DIRECTOR DEL ENSAYO
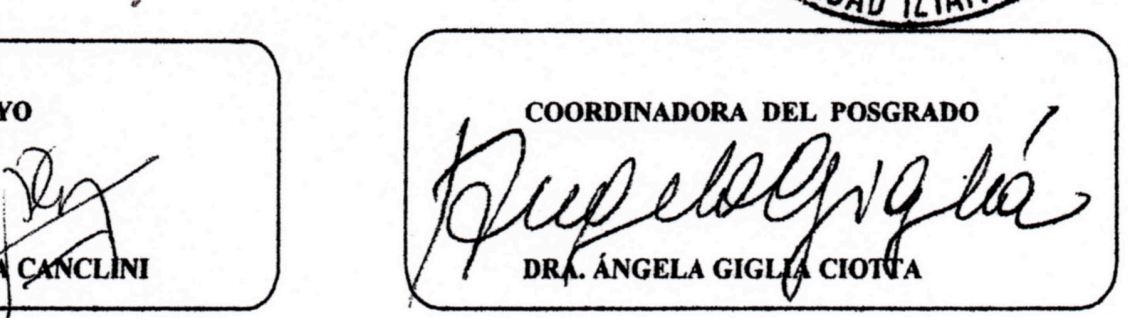

DR. NESTOR RAÚL GARCIA CANCLINI 